\title{
Expression of class II major histocompatibility complex antigens on pancreatic B cells in the NOD mouse
}

\author{
T. Hanafusa, H. Fujino-Kurihara, A. Miyazaki, K. Yamada, H. Nakajima, J. Miyagawa, N. Kono and S. Tarui \\ Second Department of Internal Medicine, Osaka University Medical School, Fukushima, Osaka, Japan
}

Summary. To elucidate the role of class II major histocompa-
tibility complex antigen expression on pancreatic B cells in
the development of diabetes in the non-obese diabetic (NOD)
mouse, indirect immunofluorescence was employed for I-A
staining on Bouin-fixed pancreas sections of NOD mice (I-A
of which was reported as d), B10.GD (I-A, d), BALB/c
(I-A, d) and C3H/He (I-A, k). I-A positive islets were ob-
served in all NOD mice examined. Positive reaction was
detected in islets both with and without lymphocytic infiltra-
tions. Double staining with anti-insulin, glucagon, somatosta-
tin or pan creatic polypeptide antibodies revealed that I-A

Mononuclear cell infiltration to pancreatic islets, namely insulitis, has been recognized as one of the main characteristics of human Type 1 (insulin-dependent) diabetes. Accumulating evidence strongly suggests that the insulitis and the following Type 1 diabetes is of autoimmune origin [1].

The non-obese diabetic (NOD) mouse develops develops insulitis after 4 weeks of age, long before the seemingly abrupt onset of ketosis-prone diabetes at 15 to 20 weeks [2]. Various reports including our own [3] indicate that the autoimmune mechanism is mainly responsible for the development of diabetes in this animal. However, what triggers the autoimmune reaction has not been studied.

Recently, the aberrant expression of class II major histocompatibility complex (MHC) antigens (DR, DQ and DP in man and I-A and I-E in mouse) on target cells in human autoimmune thyroid diseases [4] and several other disorders including Type 1 diabetes $[5,6]$ has been noted, and is hypothesized to initiate the autoimmune response against the target [7]. In the present study the aberrant expression of I-A antigen on islet cells was examined in the NOD mouse to reveal the role of this phenomenon in the pathogenesis of diabetes in this animal model. positive cells corresponded with insulin cells, while other types of pancreatic islet cells were virtually negative for I-A. Weaker staining was seen in islets of B10.GD and, to a lesser extent, in those of $\mathrm{BALB} / \mathrm{c}$ mice. $\mathrm{C} 3 \mathrm{H} / \mathrm{He}$ mouse islet cells showed no I-A expression. These results demonstrated the expression of I-A antigens on pancreatic B cells in the NOD mouse.

Key words: Class II MHC antigen, I-A antigen, NOD mouse, Type 1 diabetes, insulitis, pancreatic B cell, autoimmunity, pathogenesis, pancreatic islet.

\section{Materials and methods}

\begin{abstract}
Animals
Seventeen female NOD mice (I-A of which was reported as d) from 5 days to 24 weeks of age and 6 male NOD mice from 5 to 20 weeks of age were either purchased from Clea Japan, Inc. (Tokyo, Japan) or obtained from Dr. Y. Tochino (Shionogi Research Laboratories, Osa$\mathrm{ka}$, Japan). Table 1 shows the sex, age and the presence or absence of glycosuria in all NOD mice studied. All male mice, female animals under 14 weeks of age and one female mouse 18 weeks of age showed no glycosuria, while another female mouse 18 weeks of age and one female animal 24 weeks of age had glycosuria at the time of experiment. Six B10.GD mice (I-A, d) kindly donated by Dr. C.S. David, Mayo Clinic, Rochester, Minn, USA, six BALB/c mice (I-A, d) purchased from Shizuoka Laboratory Animal Centre, Shizuoka, Japan, and six $\mathrm{C} 3 \mathrm{H} / \mathrm{He}$ strain (I-A, k) from Charles River Laboratory, Atsugi, Japan, were used for comparison. Neither B10.GD, BALB/c nor $\mathrm{C} 3 \mathrm{H} / \mathrm{He}$ mice had glycosuria.
\end{abstract}

\section{Monoclonal antibodies}

Mouse monoclonal antibodies against I-A products were kindly donated by Dr. J.Klein (Max-Planck-Institut für Biologie, Tübingen, FRG) [8]. Clone Iam 15 reacts with I-A (b, d, j, k, kv1), Iam 25 with I-A (b, d, q, p, v, w3, w13), Iam30 with I-A (b, d, j, p, g, v, w3, w13) and Iam52 with I-A (d, f, j, v, w3, w13, w16). Mouse monoclonal antibody against human Leu7 antigen (Becton Dickinson Monoclonal Center, Inc., Mountain View, Calif, USA) was used as the irrelevant control. 
Table 1. Sex, age, glycosuria, insulitis and I-A expression of NOD mice

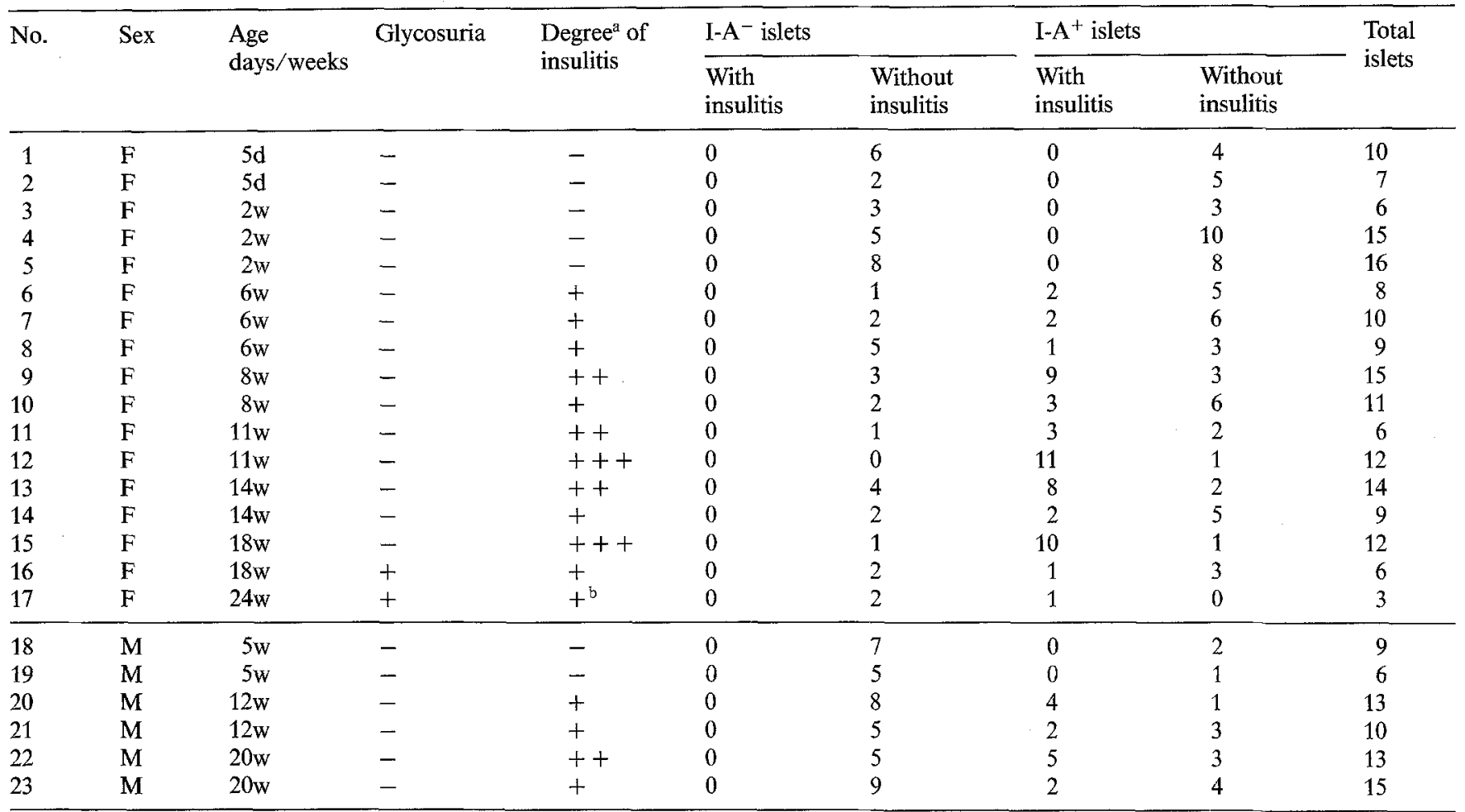

${ }^{a}(-)$ no insulitis; $(+)$ mild insulitis; $(++)$ moderate insulitis; $(+++)$ severe insulitis; ${ }^{b}$ only a few atrophic islets with mild cellular infiltration remained

Appropriate concentration of each monoclonal antibody was previously determined on thymus and spleen sections.

\section{Histological study}

Two micrometre sections were cut from Bouin-fixed pancreases of the mice. Haematoxylin and eosin staining was performed for the detection of lymphocytic infiltrations. Islets with lymphocytes around and/ or inside were judged as those with insulitis, while the ones without any lymphocytes were regarded as intact islets. The degree of insulitis was classified in each mouse by two examiners as follows; ( -$)$ no insulitis, i.e., no islet had lymphocytic infiltration; $(+)$ mild insulitis, i.e., $<30 \%$ of islets had insulitis; $(++)$ moderate insulitis, i.e., $30 \% \leqq$ islets with insulitis $<60 \%$; $(+++)$ severe insulitis, i.e., $\geqq 60 \%$ of islets had insulitis.

\section{Immunohistochemical study}

Indirect immunofluorescence was carried out for the assessment of I-A expression. Pancreas sections were first incubated for $16 \mathrm{~h}$ at $4^{\circ} \mathrm{C}$ with properly diluted mouse monoclonals. After a wash for $15 \mathrm{~min}$ with phosphate-buffered saline ( $\mathrm{pH}$ 7.4) the sections were incubated for $1 \mathrm{~h}$ at room temperature with biotinylated goat anti-mouse immunoglobulins (Igs) (E-Y Laboratories, San Mateo, Calif, USA), followed by a similar incubation with fluorescein isothiocianate (FITC)-conjugated avidin (E-Y Laboratories).

Double immunofluorescence method was employed to identify the cell type of I-A positive cells. Guinea pig anti-insulin (Otsuka Assay Laboratories, Tokushima, Japan) or rabbit anti-glucagon (donated by Dr. S. Iwasa, Takeda Pharmaceutical, Osaka, Japan), anti-somatostatin (Miles Laboratories, Elkhart, Ind, USA) or anti-bovine pancreatic polypeptide (PP) antibodies (donated by Dr. R. E. Chance, Eli Lilly \& Company, Indianapolis, Ind, USA) followed by thodamine-conjugated rabbit anti-guinea pig Igs (Zymed Laboratories Inc., San
Francisco, Calif, USA) or Texas Red-conjugated donkey anti-rabbit Igs (Amersham International, Amersham, UK), respectively, were applied on the sections which had been stained for I-A. Preliminary experiment confirmed that these conjugates cross-react with neither mouse Igs, goat Igs nor avidin. A fluorescence microscope equipped with epi-illumination (Olympus, BH-2 type, Tokyo, Japan) was used for the assessment of the reaction.

\section{Results}

\section{Histological study}

No mice 5 days and 2 weeks of age showed lymphocytic infiltration to islets, whereas the mice older than that age had various degrees of insulitis (Table 1).

\section{Immunohistochemical study}

I-A positive islet cells were detected in all 23 NOD mice. The intensity of the staining varied from islet to islet; some were completely negative. Female NOD mice tended to show a higher incidence of I-A expression in islets than males. No relationship was observed in the present study between the expression of I-A molecules and the age of the mice. I-A positive islet cells were seen on all islets with mononuclear cell infiltration and on about a half of those without infiltrates. There was no noticeable difference in the intensity of I-A staining between the islets with insulitis and those without. The expression of I-A antigens in relation to the sex, age, gly- 

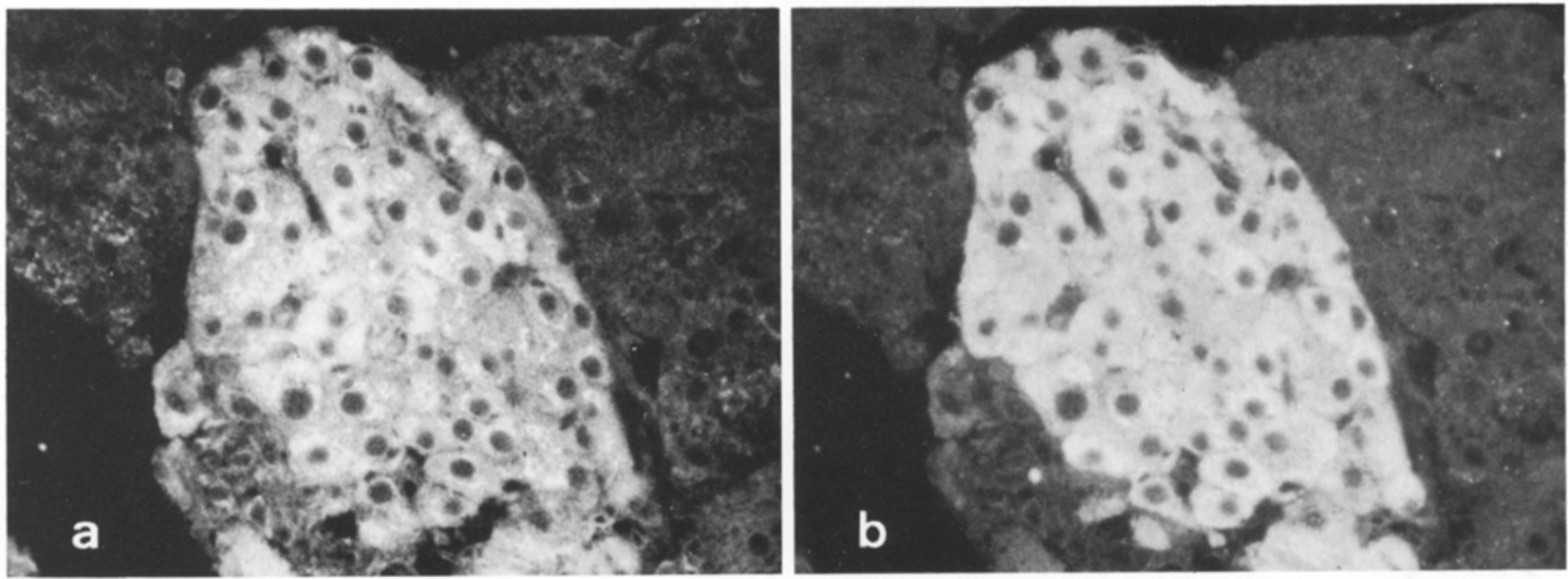

Fig. $1 \mathrm{a}$ and b. Double immunofluorescence staining of the pancreas section from an 8-week-old NOD mouse using (a) anti-I-A (Iam52) and (b) anti-insulin $(\times 400)$. Most of the islet cells were positively stained with anti-I-A; they corresponded almost completely with insulin-positive cells, indicating that I-A-positive cells are pancreatic B cells
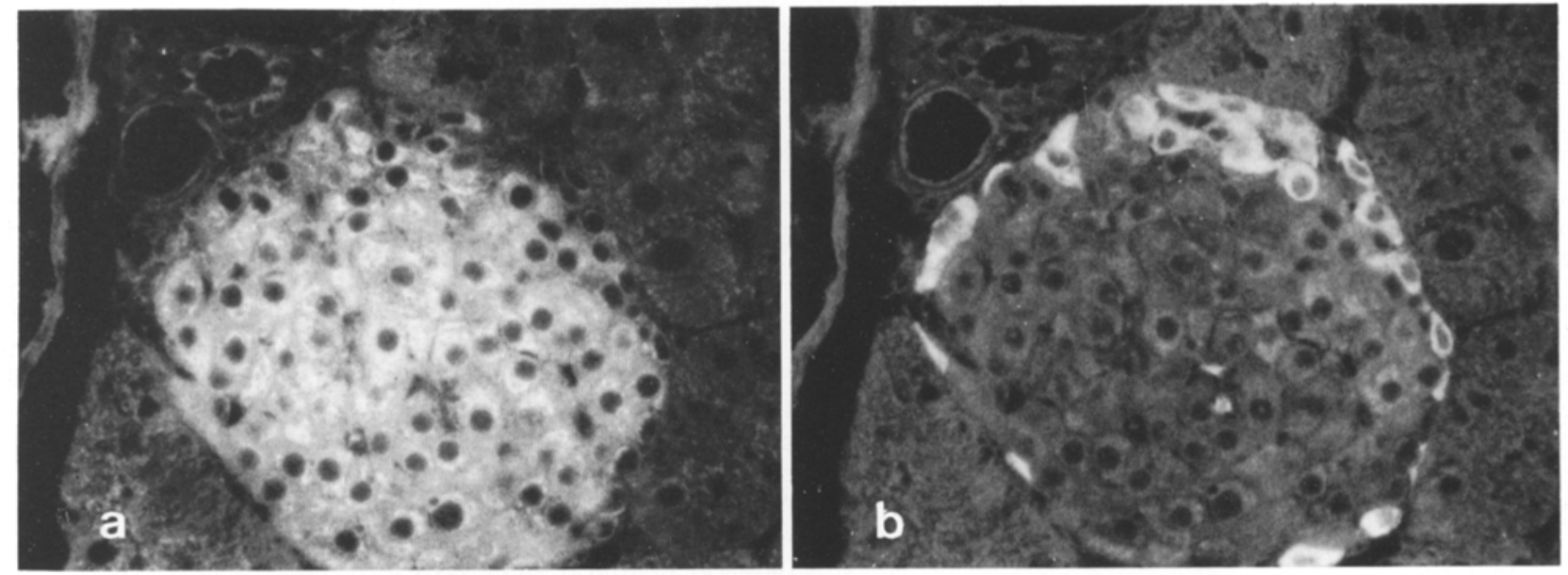

Fig. 2 a and b. Double immunofluorescence staining of the same NOD mouse shown in Figure 1, using (a) anti-I-A (Iam52) and (b) anti-glucagon $(\times 400)$. Islet cells in the upper portion of this islet were negative with anti-I-A; they reacted with anti-glucagon, indicating that A cells are I-Anegative
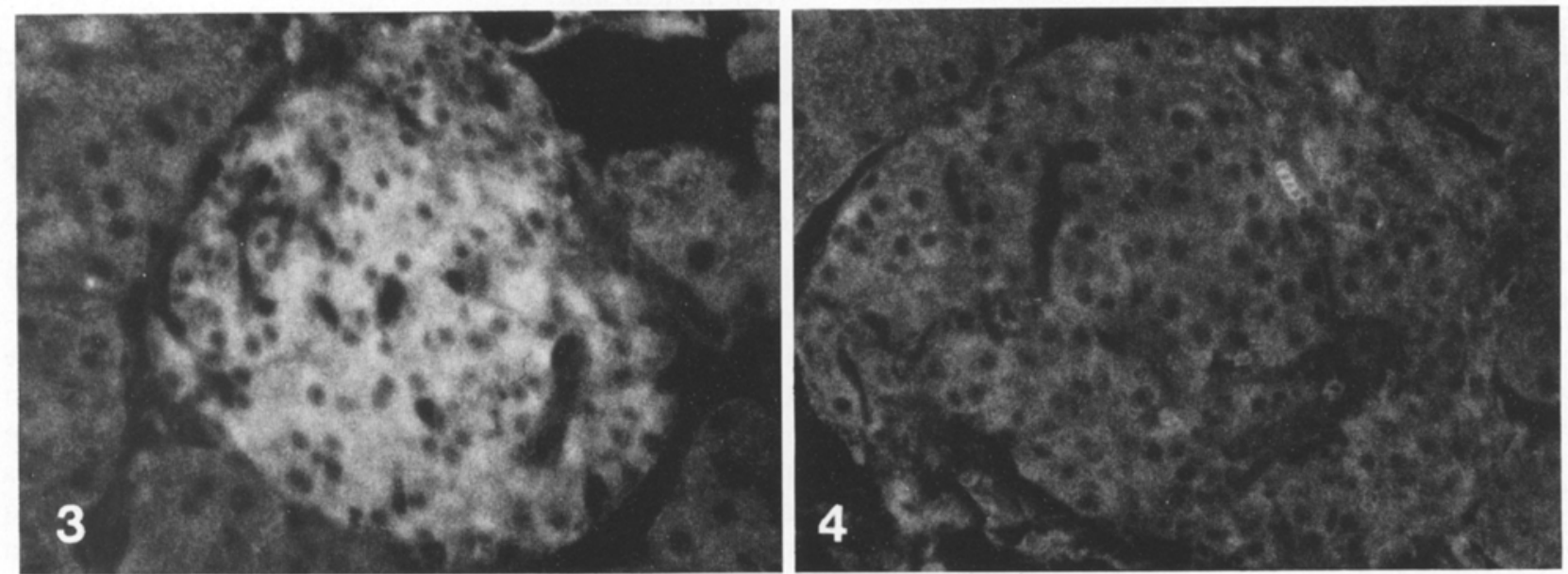

Fig.3. The pancreas section of a 10-week-old B10.GD mouse stained with anti-I-A (Iam15) $\times 400)$. Islet cells were weakly positive for I-A. The staining using Iam52 showed practically the same picture

Fig. 4. The pancreas section of an 8 -week-old $\mathrm{C} 3 \mathrm{H} / \mathrm{He}$ mouse stained with anti-I-A $(\operatorname{Iam} 15)(\times 400)$. Islet cells were negative for I-A 
cosuria and the presence of insulitis in each mouse is illustrated in Table 1. Different monoclonal antibodies gave different intensity of I-A staining on islet cells. The strongest I-A staining in NOD mouse islet cells was seen with Iam52. Other monoclonals (Iam15, Iam25 and Iam30) gave weaker staining on NOD mouse islet cells.

Double immunofluorescence study revealed that I-A positive islet cells corresponded almost completely with insulin-containing cells (Fig. 1 a, b), while glucagon (Fig. $2 \mathrm{a}, \mathrm{b}$ ), somatostatin and PP cells were virtually negative for I-A.

Weaker I-A staining was observed in the islets of B10.GD (Fig.3) and, to a lesser extent, in those of $\mathrm{BALB} / \mathrm{c}$ mice using Iam52 or Iam15. Other monoclonals (Iam25 and Iam30) gave almost negative I-A staining in the two mouse strains. $\mathrm{C} 3 \mathrm{H} / \mathrm{He}$ mouse islet cells showed no I-A expression with any monoclonals (Fig.4). Since only Iam15 is assumed to react with ' $k$ ', which is the I-A type of the $\mathrm{C} 3 \mathrm{H} / \mathrm{He}$ mouse, the difference in I-A staining between the four mouse strains could only be compared using Iam 15 . When Iam15 was used to compare the intensity of I-A staining on islet cells between different strains, NOD mice showed the strongest fluorescence, followed by B10.GD and $\mathrm{BALB} / \mathrm{c}$ mice, although the difference is not striking. On the other hand, Iam52 gave the more prominent difference between NOD, B10.GD and BALB/c mice. Thus, this monoclonal was used to compare the three strains, although this is not supposed to react with $\mathrm{C} 3 \mathrm{H} / \mathrm{He}$ mouse I-A antigen. As seen in NOD mice, I-A positive islet cells in B10.GD mice corresponded with insulin cells and were different from glucagon, somatostatin or PP cells.

\section{Discussion}

Following the first report in autoimmune thyroid diseases [4], the aberrant expression of class II MHC antigen has been revealed in various autoimmune disorders such as Type 1 diabetes $[5,6]$ and primary biliary cirrhosis [9]. In the BB rat, another animal model for insulitisassociated Type 1 diabetes, a similar finding was reported [10], suggesting that the aberrant expression of these antigens may be a common phenomenon in organ-specific autoimmune diseases. Since class II MHC molecules play a key role in the presentation of antigens and regulation of the immune response, a hypothesis has been proposed that the expression of these molecules could help to present surface autoantigens to helper $T$ cells to initiate the autoimmune response against the autoantigens [7]. In fact, DR antigens induced on thyroid cells were able to present a peptide $\mathrm{p} 20$ derived from influenza haemagglutinin to stimulate HLA-DR-matched cloned influenza-specific $T$ cells [11]. Thus, it is reasonable to speculate that class II MHC antigens on pancreatic $B$ cells in the spontaneously diabetic NOD mouse demonstrated in this study could trigger a chain of autoimmune reactions against themselves.

It is worth noting that the aberrant expression of I-A antigens was observed even on islets without any lymphocytic infiltration, suggesting that the expression of these molecules is not a secondary phenomenon induced by infiltrating lymphocytes but precedes the invasion of them. Also interesting is that the incidence of I-A positive islets tended to be higher in female NOD mice than that in males. This observation may well be relevant to the lower incidence of diabetes in male NOD mice, but further study is needed to confirm the relationship between the I-A expression and the incidence of diabetes.

Another important point in the present study is that the expression of I-A antigen on pancreatic $B$ cells was observed in B10.GD and BALB/c mice, both of which are not prone to diabetes, as well as in insulitis-associated NOD mice. Three explanations could be possible for this observation. One is that the expression of I-A antigen on target cells alone is not sufficient to initiate a chain of autoimmune reactions against the target cells, and that another factor, probably on the side of immunocytes, is needed for the full activation of autoreactive helper T cells. Another feasible explanation is that the expression of I-A antigen on islet B cells in B10.GD or $\mathrm{BALB} / \mathrm{c}$ mice is, compared with that in NOD mice, too weak to activate helper $T$ cells. The third possibility is that not the whole I-A molecule, but one glycoprotein chain or a part of it, is expressed on B cells in B10.GD or BALB/c mice. This incomplete molecule is not likely to help presenting autoantigens.

In conclusion, the present study demonstrated the expression of I-A antigen on pancreatic $B$ cells in the NOD mouse. Whether this phenomenon is closely related to the pathogenesis of insulitis and the following development of diabetes needs further investigation.

Acknowledgements. We wish to thank Dr. Y. Tochino (Shionogi Research Laboratories, Osaka, Japan) for providing us with the NOD mice, Dr. C.S.David (Mayo Clinic, Rochester, Minn, USA) for B10.GD mice, Dr. J. Klein (Max-Planck-Institut für Biologie, Tübingen, FRG) for monoclonal antibodies against I-A antigens, Dr. S. Iwasa (Takeda Pharmaceutical, Osaka, Japan) for anti-glucagon antibody, and Dr. R. E. Chance (Eli Lilly \& Company, Indianapolis, Ind, USA) for anti-bovine pancreatic polypeptide antibody. We are also indebted to Professor K. Nonaka (Kurume University, Kurume, Japan) for his continuous encouragement. This work was supported by the Scientific Research Fund (No.60570532) from the Ministry of Education, Science and Culture of Japan, grants from the Mochida Memorial Foundation for Medical and Pharmaceutical Research and Yamanouchi Foundation for Research on Metabolic Disorders in Japan.

\section{References}

1. Bottazzo GF (1984) $\beta$-Cell damage in diabetic insulitis: are we approaching a solution? Diabetologia 26: 241-249

2. Makino S, Kunimoto K, Muraoka T, Mizushima Y, Katagiri K, Tochino $Y(1980)$ Breeding of a non-obese diabetic strain of mice. Exp Anim 29:1-13 
3. Miyazaki A, Hanafusa T, Yamada K, Miyagawa J, Fujino-Kurihara H, Nakajima H, Nonaka K, Tarui S (1985) Predominance of T lymphocytes in pancreatic islets and spleen of pre-diabetic nonobese diabetic (NOD) mice: a longitudinal study. Clin Exp Immunol 60: 622-630

4. Hanafusa T, Pujol-Borrell R, Chiovato L, Russell RCG, Doniach D, Bottazzo GF (1983) Aberrant expression of HLA-DR antigen on thyrocytes in Graves' disease: relevance for autoimmunity. Lancet 2: 1111-1115

5. Bottazzo GF, Dean BM, McNally JM, MacKay EH, Swift PGF, Gamble DR (1985) In situ characterization of autoimmune phenomena and expression of HLA molecules in the pancreas in diabetic insulitis. N Engl J Med 313: 353-360

6. Foulis AK, Farquharson MA (1986) Aberrant expression of HLADR antigens by insulin-containing $\beta$-cells in recent onset type I diabetes mellitus. Diabetes 35: 1215-1224

7. Bottazzo GF, Pujol-Borrell R, Hanafusa T, Feldmann M (1983) Role of aberrant HLA-DR expression and antigen presentation in induction of endocrine autoimmunity. Lancet 2:1115-1118

8. Klein J, Figueroa F, David CS (1983) H-2 Haplotypes, genes and antigens: second listing. II. The H-2 complex. Immunogenetics $17: 553-596$
9. Ballardini G, Mirakian R, Bianchi FB, Pisi E, Doniach D, Bottazzo GF (1984) Aberrant expression of HLA-DR antigens on bileduct epithelium in primary biliary cirrhosis: relevance to pathogenesis. Lancet 2: 1009-1013

10. Dean BM, Walker R, Bone AJ, Baird JD, Cooke A (1985) Prediabetic $\mathrm{BB} / \mathrm{E}$ rat: lymphocyte subpopulations in the pancreatic infiltrate and expression of rat MHC class II molecules in endocrine cells. Diabetologia 28: 464-466

11. Londei M, Lamb JR, Bottazzo GF, Feldmann M (1984) Epithelial cells expressing aberrant MHC Class II determinants can present antigen to cloned human T lymphocytes. Nature 312: 639-641

Received: 26 June 1986

and in revised form: 30 December 1986

Dr. T. Hanafusa

Second Department of Internal Medicine

Osaka University Medical School

Fukushima, Osaka 553

Japan 